\title{
MICROFILTRACIÓN EN RESTAURACIONES PARCIALES INDIRECTAS CEMENTADAS CON CEMENTOS RESINOSOS DUALES AUTOADHESIVOS
}

\author{
MICROLEAKAGE IN INDIRECT PARTIAL RESTORATIONS CEMENTED WITH \\ SELF-ADHESIVE DUAL RESIN CEMENTS \\ Javier Cleto Zaga Bendezú \\ jzagab@hotmail.com \\ ORCID: 0000-0001-6732-9423 \\ Ana Isabel López-Flores \\ ailopez@ucientifica.edu.pe \\ ORCID 0000-0002-1104-2439
}

\section{RESUMEN}

El objetivo de este estudio es comparar in vitro el grado de microfiltración marginal en incrustaciones de cerámica inyectada realizadas con dos cementos resinosos duales autoadhesivos seT PP (SDI) y RelyX U200 (3M ESPE). Se evaluaron 40 premolares superiores e inferiores extraídos por motivos ortodónticos y se dividieron en dos grupos según cemento asignado $(n=20)$. Luego, se realizó la preparación de cavidades tipo clase II, de aproximadamente $3 \mathrm{~mm}$ de ancho en la caja oclusal y proximal; y $2 \mathrm{~mm}$ de espesor en el piso gingival y la pared oclusal. Se restauraron de manera indirecta con restauraciones inyectadas de cerámica IPS e.max Press (Ivoclar Vivadent), se cementaron con los cementos estudiados según las especificaciones del fabricante. Los especímenes fueron guardados en un frasco y llevados a termociclado; luego, se realizaron cortes transversales a las coronas y se evaluó el grado de microfiltración por medio de un estereoscopio. Al comparar la microfiltración entre el cemento seT PP y RelyX U200, se concluyó que no existe una asociación estadísticamente significativa entre el grado de microfiltración y el tipo de cemento (prueba de Chi-cuadrado de Pearson; $p>0,05)$ y al observar el grado de microfiltración del seT PP y el RelyX U200 en la cementación de incrustaciones cerámicas inyectadas IPS e.max Press. En conclusión, no se hallaron diferencias significativas entre los dos cementos resinosos autoadhesivos. En ambos prevaleció la no microfiltración; sin embargo, para seT PP, la microfiltración fue del $17,5 \%$ en $1 / 3$ de la pared cervical.

Palabras clave: microfiltración, cerámica, cementos resinosos

\section{ABSTRACT}

Objective: To compare in vitro the degree of marginal microleakage in injected embedded ceramics cemented with two self-adhesive dual resin cements: seT PP (SDI) and RelyX U200 (3M ESPE). Materials and methods: 40 upper and lower premolars extracted for orthodontic

Citar como: Zaga Bendezú J y López-Flores A. Microfiltración en restauraciones parciales indirectas cementadas con cementos resinosos duales autoadhesivos. Rev Cient Odontol (Lima). 2019; 7 (2): 33-41. 
reasons were evaluated and divided into two groups according to the cement assigned $(n=20)$. The preparation of class II cavities was approximately 3 $\mathrm{mm}$ wide in occlusal and proximal boxes and $2 \mathrm{~mm}$ thick on the gingival floor and the occlusal wall. They were indirectly restored with restorations injected with IPS e.max Press ceramic (Ivoclar Vivadent), cemented with one of the study cements following the manufacturer's specifications. The samples were stored in a bottle and thermocycled. Cross sections of the crowns were then made, and the degree of microleakage was evaluated by stereoscopy. Results: There was no statistically significant association between the degree of microleakage and the type of cement used (Pearson's Chisquare Test; $p>0.05$ ) or the degree of microfiltration of seT PP and RelyX U200 in IPS e.max Press-injected ceramic cementation. No microleakage prevailed with both cements; however, seT PP showed $17.5 \%$ microleakage in $1 / 3$ of the cervical wall. Conclusions: No significant differences were observed between the two self-adhesive resinous cements in relation to microleakage.

Keywords: microleakage, ceramics, resin cements

\section{INTRODUCCIÓN}

El avance de los materiales y técnicas de restauración continúa mejorando el éxito clínico de numerosos procedimientos de restauración. A pesar de estas innovaciones, la microfiltración persiste como una de las principales causas del fracaso de la restauración $\left({ }^{1,2}\right)$. La microfiltración es el movimiento de bacterias, fluidos, moléculas o iones entre el diente y los márgenes de restauración; asimismo, es el resultado de la invasión del ambiente externo a través de los márgenes de la restauración, que también puede ocurrir internamente $\left({ }^{1}\right)$.
La microfiltración puede causar una variedad de efectos adversos, como caries secundaria, mayor sensibilidad del diente restaurado y tinción interfacial que conduce a la patología pulpar $\left(^{3}\right)$. Esta ocurre más comúnmente cuando el margen gingival de cualquier restauración se coloca debajo de la unión cemento-esmalte, porque la unión a la dentina es menos predecible que el esmalte debido a su patrón complejo y menor contenido mineral $(3,4)$.

Los cementos resinosos autoadhesivos, también conocidos como todo en uno, tienen una buena resistencia de adhesión a la dentina, el esmalte y las porcelanas sin la necesidad de agentes de unión separados. Estos cementos se pueden unir a una superficie dental no tratada que no ha sido microabrasada o pretratada con un agente grabador o agente de unión; así, la cementación se realiza en un solo paso. Estos cementos contienen ácido fosfórico que se injerta en la resina. Una vez que se inicia la mezcla, el ácido fosfórico reacciona con las partículas de relleno y la dentina en presencia de agua, lo que forma un enlace. La resina se polimeriza en un polímero reticulado, como es el caso de la unión de resina compuesta $(5,6)$.

El éxito del tratamiento restaurador no depende solo del profesional, sino también de una suma de factores, así como del material utilizado y el paciente. Existen diversos materiales que favorecen la estética y mantienen la función, tales como la porcelana, los cerómeros y las resinas, al igual que materiales cementantes que ofrecen optimizar el sellado marginal, la longevidad de la restauración y la viabilidad del diente huésped $\left({ }^{7}\right)$. El agente de cementación es vital en este proceso, por lo cual debe cumplir con ciertos requisitos como biocompatibilidad, baja viscosidad y resistencia mecánica, adhesión al diente y a la restauración, baja solubilidad, 
estabilidad de color en el tiempo, radiopacidad, fácil manipulación, entre otros. Por lo tanto, el propósito del presente estudio fue comparar in vitro el grado de microfiltración marginal en restauraciones parciales indirectas de cerámica inyectada cementadas con dos cementos resinosos duales autoadhesivos.

\section{MATERIALES Y MÉTODOS}

El presente estudio fue aprobado por el comité de Ética de la Facultad de Odontología de la Universidad Científica del Sur, con el código de aprobación EE-REG.002.V. Para el cálculo del tamaño muestral se utilizó la fórmula de comparación de dos proporciones y se obtuvo un total de 20 especímenes por grupo $(\mathrm{N}=40)$. Los criterios de inclusión incluían especímenes sin caries, fracturas ni grietas. Estos, una vez seleccionados, fueron distribuidos en dos grupos según el cemento asignado: seT PP $(\mathrm{n}=$ 20) y RelyX U200 $(n=20)$. Se realizaron cavidades Clase II de aproximadamente $3 \mathrm{~mm}$ de ancho tanto en la caja oclusal como en proximal, y $2 \mathrm{~mm}$ de espesor tanto en el piso gingival como en la pared oclusal. Se procedió a tomar modelos a cada una de las piezas para, posteriormente, confeccionar los patrones de cera de las incrustaciones a fin de procesarlas e inyectar cerámica IPS e.max Press.

Para realizar el proceso de cementación en ambos grupos, se trató la cara interna de cada incrustación, se introdujo en un vaso dappen con alcohol durante unos segundos, se secó con aire de la jeringa triple y se trató la superficie interna con ácido fluorhídrico al $10 \%$ durante 20 segundos, lavar y secar, y se aplicó ácido ortofosfórico al 37\%, durante 30 segundos. Luego, se lavó con agua de la jeringa triple profusamente y se secó con aire. Se trató con silano por 2 minutos y se limpió con escobilla de profilaxis, para luego aplicar el cemento respectivo generosamente y se retiraron los excesos del material cementante con un cepillo minibrush. Se fotopolimerizó mediante una lámpara con una potencia de $1,470 \mathrm{~mW} / \mathrm{cm}^{2}$, durante 30 segundos, por proximal y oclusal, para dejar almacenadas las piezas en solución salina durante 24 horas. Finalmente, se termocicló a 1000 ciclos de 5 min a $5^{\circ} \mathrm{C}, 25 \mathrm{~min}$ a $37^{\circ} \mathrm{C}, 5 \mathrm{~min}$ a $55^{\circ} \mathrm{C}, 25 \mathrm{~min}$ a $37^{\circ} \mathrm{C}$.

La parte radicular se aisló con resina acrílica y se dejó expuesta solo la parte coronal del diente para colocarlo en una solución de azul de metileno al 1\%, durante 24 horas. Pasado este tiempo, se lavaron las piezas y se secó al aire. Con un disco diamantado y un micromotor se realizaron cortes transversales a las coronas de las muestras, siempre pasando por la parte media de las incrustaciones de ambos grupos, a fin de poder analizar el porcentaje de microfiltración. El corte se realizó de forma intermitente para disipar el calor producido. Finalmente, las muestras fueron observadas en un microscopio estereoscópico marca Olympus por un operador entrenado, con un aumento de 10X. Se determinó la distancia de la penetración del colorante en la interfase diente-restauración y se categorizó de la siguiente manera: 0 : no filtración; 1: penetración hasta 1/3 de la pared cervical; 2: penetración hasta 2/3 de la pared cervical; 3: penetración en toda la pared cervical y 4: colorante hasta la pared axial $\left(^{8}\right)$.

Los datos fueron analizados usando el programa estadístico SPSS (IBM, EE. UU.) versión 23. Se utilizó un nivel de significancia del 5\%. Las variables cualitativas fueron analizadas mediante estadígrafos tales como frecuencias y porcentajes, y para la estadística analítica y el establecimiento de relaciones entre las variables se utilizó la prueba de Chi-cuadrado. Se asumió una asociación estadísticamente significativa cuando el resultado fue de $p<0,05$. 


\section{RESULTADOS}

En la tabla 1 se observa el grado de microfiltración del SET PP de SDI, en la cementación de restauraciones parciales indirectas de cerámicas inyectadas IPS e.max Press. Se obtuvo una mayor prevalencia de no filtración, con un $40 \%$, seguida por un $35 \%$ de penetración hasta $1 / 3$ de la pared cervical y un $20 \%$ de penetración hasta $2 / 3$ de la pared cervical.

En la tabla 2 se observa el grado de microfiltración marginal del cemento Rely X U 200 3M ESPE en la cementación de restauraciones parciales indirectas de cerámicas inyectadas IPS e.max Press. Se obtuvo una mayor prevalencia de no filtración, con un $55 \%$, seguida por un $20 \%$ de penetración en toda la pared cervical y un $10 \%$ de penetración hasta $2 / 3$ de la pared cervical y $1 / 3$ de la pared cervical, respectivamente.
En la tabla 3 se observa la comparación del grado de microfiltración marginal en restauraciones parciales indirectas de cerámica inyectada, cementadas con dos cementos resinosos duales autoadhesivos a nivel del margen cervical. El cemento SET presentó una filtración del $20 \%$ y una penetración hasta $1 / 3$ de la pared cervical del $17,5 \%$, mientras que el cemento RelyX U200 presentó una no filtración del $27.5 \%$ y una penetración en toda la pared cervical del $10 \%$. No hay asociación estadísticamente significativa entre el grado de microfiltración marginal en restauraciones parciales indirectas de cerámica inyectada cementadas con dos cementos resinosos duales autoadhesivos (prueba de Chi-cuadrado: $p>0,05$ ).

\section{DISCUSIÓN}

En la actualidad, no se han encontrado estudios previos que comparen la

Tabla 1. Grado de microfiltración del SET PP de SDI en la Cementación de Restauraciones PARCIALES INDIRECTAS DE CERÁMICAS INYECTADAS IPS E.MAX PRESS

\begin{tabular}{|l|c|c|}
\hline Grado de microfiltración & SET & $\%$ \\
\hline No filtración & 8 & $40,0 \%$ \\
\hline Penetración hasta $1 / 3$ de la pared cervical & 7 & $35,0 \%$ \\
\hline Penetración hasta $2 / 3$ de la pared cervical & 4 & $20,0 \%$ \\
\hline Penetración en toda la pared cervical & 0 & $0,0 \%$ \\
\hline Colorante hasta la pared pulpar & 1 & $5,0 \%$ \\
\hline
\end{tabular}

Tabla 2. Grado de microfiltración marginal del cemento Rely X U 200 3M ESPE en la CEMENTACIÓN DE RESTAURACIONES PARCIALES INDIRECTAS DE CERÁMICAS INYECTADAS IPS E.MAX PRESS

\begin{tabular}{|l|c|c|}
\hline \multirow{2}{*}{ Grado de microfiltración } & \multicolumn{3}{|c|}{ RelyX U200 } \\
\hline No filtración & $n$ & $\%$ \\
\hline Penetración hasta 1/3 de la pared cervical & 2 & $55,0 \%$ \\
\hline Penetración hasta $2 / 3$ de la pared cervical & 2 & $10,0 \%$ \\
\hline Penetración en toda la pared cervical & 4 & $10,0 \%$ \\
\hline Colorante hasta la pared pulpar & 1 & $5,0 \%$ \\
\hline
\end{tabular}


TABLA 3. COMPARACIÓN DEL GRADO DE MICROFILTRACIÓN MARGINAL EN RESTAURACIONES PARCIALES INDIRECTAS DE CERÁMICA INYECTADA CEMENTADAS CON DOS CEMENTOS RESINOSOS DUALES AUTOADHESIVOS, A NIVEL DEL MARGEN CERVICAL

\begin{tabular}{|c|c|c|c|c|c|c|c|}
\hline & & $\begin{array}{l}\text { No } \\
\text { filtración }\end{array}$ & $\begin{array}{l}\text { Penetración } \\
\text { hasta } 1 / 3 \\
\text { de la pared } \\
\text { cervical }\end{array}$ & $\begin{array}{l}\text { Penetración } \\
\text { hasta } 2 / 3 \\
\text { de la pared } \\
\text { cervical }\end{array}$ & $\begin{array}{l}\text { Penetración } \\
\text { en toda } \\
\text { la pared } \\
\text { cervical }\end{array}$ & $\begin{array}{l}\text { Colorante } \\
\text { hasta la } \\
\text { pared } \\
\text { pulpar }\end{array}$ & Valor $p$ \\
\hline \multirow{2}{*}{ SET } & $\mathrm{n}$ & 8 & 7 & 4 & 0 & 1 & \multirow{4}{*}{0,095} \\
\hline & $\%$ & $20,0 \%$ & $17,5 \%$ & $10,0 \%$ & $0,0 \%$ & $2,5 \%$ & \\
\hline \multirow{2}{*}{ RelyX U200 } & $n$ & 11 & 2 & 2 & 4 & 1 & \\
\hline & $\%$ & $27,5 \%$ & $5,0 \%$ & $5,0 \%$ & $10,0 \%$ & $2,5 \%$ & \\
\hline
\end{tabular}

*Prueba de Chi-cuadrado de Pearson; $p<0,05$

microfiltración en preparaciones parciales indirectas inyectadas usando los agentes cementantes seT PP de SDI y RelyX ${ }^{\mathrm{TM}}$ U200 (3M ESPE), mediante la evaluación de diferentes superficies. Por lo tanto, este estudio brindará a los profesionales estomatólogos más información y evidencias científicas al respecto. Se tuvo como propósito comparar in vitro el grado de microfiltración marginal en restauraciones parciales indirectas de cerámica inyectada, cementadas con dos cementos resinosos duales autoadhesivos. La muestra utilizada fueron 40 especímenes distribuidos en dos grupos, según cemento asignado.

En este estudio la población estuvo conformada por primeros premolares maxilares y mandibulares extraídos por motivos ortodónticos, al igual que Barbosa $\left({ }^{9}\right)$, Trindade $\left({ }^{10}\right)$ y Saeid $\left({ }^{11}\right)$, que usaron las mismas piezas dentales para sus estudios. Por otra parte, hubo un grupo que realizó su investigación en terceras molares (Buchelli [ $\left.{ }^{12}\right]$, Otavo $\left[{ }^{13}\right]$ y Mejía $\left.\left[{ }^{14}\right]\right)$, casos en los que no se indica cuál fue el motivo de elección de sus piezas dentarias. Taschner $\left({ }^{15}\right)$, a diferencia de los demás estudios, usó premolares y molares en boca, lo cual le permitió observar la microfiltración en piezas dentales que estaban expuestas a diferentes condiciones bucales (como saliva y masticación).
La microfiltración en piezas dentarias se evalúa mayormente con estudios in vitro y de esa manera se determinan las propiedades de sellado de los diferentes materiales. Entre las técnicas más comunes se encuentra la microfiltración de moléculas bacterianas, trazadores químicos, radioactivos y penetración de tintes, las cuales proporcionan cierta información sobre el posible comportamiento de estos materiales ante la evaluación con microscopia electrónica $\left({ }^{9}\right)$. En este estudio, al igual que Barboza $\left({ }^{9}\right)$ y Otavo $\left({ }^{13}\right)$, se usó la tinción azul de metileno, a diferencia de Buchelli $\left({ }^{12}\right)$, Pinos $\left({ }^{16}\right)$ y Uludag $\left({ }^{17}\right)$, quienes emplearon la tinción fucsia.

El termociclado es uno de los procedimientos que también se usan para los estudios in vitro, y por el cual las piezas dentarias son ingresadas a una máquina con diferentes ciclos, temperatura y tiempos de inmersión, lo que depende del investigador. Barboza $\left({ }^{9}\right)$ utilizó 3000 ciclos para su estudio, mientras que Otavo $\left({ }^{13}\right)$ y esta investigación usaron una cantidad menor de ciclos (1000). Las consideraciones para la elección de la cantidad de ciclos fueron determinadas por el criterio del encargado del laboratorio. Pinos $\left({ }^{16}\right)$ usó ciclos más bajos (500); en cambio, Korkut $\left({ }^{18}\right)$ y Buchelli $\left({ }^{12}\right)$ utilizaron una cantidad más elevada de ciclos (5000 y 10 000). 
Todas las investigaciones usaron un rango muy parecido de temperatura. Casi todos los especímenes in vitro de los diferentes estudios fueron evaluados con estereoscopio, el cual tiene la capacidad de mostrar los objetos en forma ampliada y tridimensional. Lo que ha variado en algunos estudios es la cantidad de aumento que han utilizado. Barboza $\left({ }^{9}\right)$ utilizó un aumento de 40x y en este estudio se empleó un aumento de 10x; los demás estudios no especificaron la cantidad de aumento que usaron. Usar un aumento alto permite una mayor amplitud de las imágenes y, por ende, una visión más exacta de la microfiltración en las piezas. Por su parte, Buchelli $\left({ }^{12}\right)$ no utilizó el mismo método, sino que observó las piezas dentarias por medio de fotografías tomadas con un lente macro de $100 \mathrm{~mm}$.

Los agentes cementantes son quienes se encargan de permitir la unión del diente con el material restaurador. Todas las piezas restauradas presentan, de una $\mathrm{u}$ otra manera, microfiltraciones. Para las incrustaciones cementadas con seT $\mathrm{PP}$, se presentó una mayor presencia de microfiltración en $1 / 3$ de la pared cervical en un $35 \%$ (7 piezas). Le siguieron $2 / 3$ de la pared cervical con un $20 \%$ (4 piezas); a nivel de pared pulpar o axial, con un $5 \%$ (1 pieza), y la no filtración figura con un $40 \%$ (8 piezas). Las microfiltraciones presentadas con este material se pudieron deber a que no tuvieron un buen sellado en algunos especímenes.

Para las incrustaciones cementadas con RelyXTM U200 (3M ESPE), un 20\% (4 piezas) presentó mayor filtración; un 10\% (2 piezas) presentó filtración en toda la pared cervical; otro 10\% (2 piezas) hasta $2 / 3$ de la pared cervical; un 5\% (1 pieza) hasta $1 / 3$ de la pared cervical, a nivel de pared pulpar o axial; y no hubo filtración en un 55\% (11 piezas). Este material presentó menos cantidad de microfiltración y a niveles más superficiales, con lo que se puede decir que tuvo un mejor sellado en comparación con el otro cemento estudiado. Los cementos seT PP de SDI y RelyX'TM U200 (3M ESPE), a nivel del margen cervical, presentaron varios especímenes sin microfiltración, lo cual se pudo deber a que el sellado fue mejor a nivel de ese margen. Con todo lo descrito, se puede deducir que en su mayoría de casos siempre se presenta un grado de microfiltración, son muy pocas las estadísticas que no presentan ninguna. Estos datos estadísticos no se han podido comparar, ya que no existe ningún estudio parecido que utilice los mismos agentes cementantes y que divida el diente de la misma manera.

Barbosa $\left({ }^{9}\right)$ comparó el grado de microfiltración en incrustaciones inlay de disilicato de litio y las cementó con resina dual de grabado total (Variolink N) y resina dual autoadhesiva (Multilink $S$ ), tras lo cual halló una diferencia estadísticamente significativa en la menor microfiltración para Variolink $\mathrm{N}$ (grabado total). Se puede decir que en este estudio no se hallaron diferencias estadísticamente significativas debido que los dos cementos resinosos fueron del mismo tipo autoadhesivo.

Pinos $\left({ }^{16}\right)$ evaluó el grado de microfiltración de incrustaciones de dióxido de zirconio (un tipo de cerámica que no usa una base vítrea de sílice) cementandas con dos tipos de cementos resinosos, uno de los cuales fue el RelyX ${ }^{\mathrm{TM}}$ U200, el mismo que se utilizó en este estudio, pero lo comparó con otro tipo de cemento, el Multilink N, y tampoco encontró diferencias estadísticamente significativas. Lo que si observó al estudiar individualmente cada cemento es que el Rely $X^{\mathrm{TM}}$ U200 presenta una mayor microfiltración que el Multilink $\mathrm{N}$ y esto se puede deber a que el cemento RelyX ${ }^{\mathrm{TM}}$ U200 es dual autoadhesivo todo en uno, lo que haría que no produzca un sellado más exacto. 
Buchelli $\left({ }^{12}\right)$ usó el mismo cemento resinoso que este estudio RelyX ${ }^{\mathrm{TM}}$ U200, pero cementando carillas de cerómeros. Al compararlo con su otro cemento, el RelyX'M ARC, observó que el primero presentaba una mayor filtración. Saeid $\left({ }^{11}\right)$ también comparó, como esta investigación, diferentes agentes cementantes resinosos, pero de otras marcas (Panavia F2, Maxcem y Biscem), y sí encontró una diferencia estadística significativa, siendo superior el cemento Panavia F2. Uludag $\left({ }^{17}\right)$ comparó igualmente distintos agentes cementantes, que fueron Panavia 21 (en una presentación diferente a la que utilizó Saeid), RelyX ${ }^{\mathrm{TM}}$ ARC y Variolink II, siendo la primera la que mostró microfiltraciones más altas. Esto se puede deber al cambio de presentación o a que los agentes cementantes con los cuales la comparó Uludag son superiores.

Con relación a la hipótesis planteada, se acepta la hipótesis nula, ya que no se encontró asociación estadísticamente significativa entre el grado de microfiltración a nivel del margen oclusal ni el cervical y el tipo de cemento resinoso dual autoadhesivo (prueba de Chicuadrado de Pearson: $p>0,05)$. Tampoco se hallaron otros estudios para realizar la comparación, lo que si se encontró fueron comparaciones con otros cementos. Se puede concluir, de manera general, que ambos cementos tendrían el mismo comportamiento clínico siempre que se sigan los protocolos de cementación establecidos y de esta manera se podría controlar la microfiltración. Esta información es de vital importancia no solo para el odontólogo, sino también para que la permanencia de las restauraciones sea más duradera en bienestar de la salud bucal de los pacientes.

\section{CONCLUSIONES}

1. La cementación con el cemento resinoso autoadhesivo SET PP de SDI en restauraciones parciales indirectas de cerámicas inyectadas con IPS. E.max Press presentó microfiltraciones en un $60 \%$.

2. La cementación con el cemento resinoso autoadhesivo RelyX U200 (3M ESPE) en restauraciones parciales indirectas de cerámicas inyectadas con IPS. E.max Press presentó microfiltraciones en un $45 \%$.

3. La cementación con los cementos resinosos autoadhesivos SET PP de SDI y RelyX U200 (3M ESPE) no presentó diferencias significativas en función a la microfiltración a nivel del margen cervical.

Contribución del autor: Javier Cleto Zaga Bendezú y Ana López-Flores han participado en la concepción del artículo, la recolección de datos, su redacción y aprobación de la versión final.

Fuente de financiamiento: Autofinanciado.

Conflictos de interés: El autor declara no tener conflictos de interés de ningún tipo. 


\section{REFERENCIAS BIBLIOGRÁFICAS}

1. AlHabdan AA. Review of microleakage evaluation tools. J Int Oral Health. 2017; 9 (4): 141-5.

2. Goldstein RE, Lamba S, Lawson NC, Beck P, Oster RA, Burgess JO, et al. Microleakage around Class $\mathrm{V}$ composite restorations after ultrasonic scaling and sonic toothbrushing around their margin. J Esthet Restor Dent. 2017; 29 (4): 41-8.

3. Gupta A, Tavane P, Gupta PK, Tejolatha B, Lakhani AA, Tiwari R, et al. Evaluation of microleakage with total etch, self etch and universal adhesive systems in Class V restorations: An in vitro study. J Clin Diagn Res. 2017; 11 (4): ZC53-6.

4. Mali PA, Deshpande S, Singh A. Microleakage of restorative materials: An in vitro study. J Indian Soc Pedod Prev Dent. 2006; 24 (1): 15-8.

5. Ascurra L, Saravia M. Efecto del tiempo de grabado con ácido fosfórico al $37,5 \%$ sobre superficie de esmalte haciendo uso de agentes cementantes autoadhesivos. Estudio in vitro. Rev Cient Odont. 2015; 3 (1): 256-64.

6. Stamatacos C, Simon J. Cementation of indirect restorations: an overview of resin cements. Compend Contin Educ Dent. 2013; 34 (1): 42-6.

7. Pilo R, Folkman M, Arieli A, Levartovsky S. Marginal fit and retention strength of zirconia crowns cemented by self-adhesive resin cements. Oper Dent. 2018; 43 (2): 151-61.

8. Kader M, Altheeb A, Al-Asmry A, Luqman M. Microleakage evaluation of class I composite restoration with incremental and bulk fill technique. J Dent Res Rev. 2015; 2 (4): 153-5.

9. Barbosa A, Espinosa C, Ortiz Y, Cuéllar M, Parra D. Microfiltración en incrustaciones inlay en disilicato de litio técnica inyectada con dos tipos de cementos resinosos. Journal Odont Col. 2016; 9 (17): 8-25.

10. Trindade F. Ceramic inlays: effect of mechanical cycling and ceramic type on restoration-dentin bond strength. Oper Dent. 2016; 41 (4): E102-17.

11. Saeid N, Saeede Z, Seyedeh M, Aye E. Comparison of the effect of Panavia F2, biscem and maxcem on microleakage of ceramic inlays: an in-vitro study. Sch $\mathrm{J}$ Dent Sci. 2015; 2 (1): 69-73.

12. Bucheli, M. Evaluación de la microfiltración en restauraciones indirectas cementadas con resina precalentada, cemento de grabado total y un agente autoadhesivo. [Tesis para especialista en Rehabilitación Oral]. Quito: Universidad San Francisco de Quito; 2017.

13. Otavo W, Velásquez L, Hernández M, Montañez G. comparación del grado de microfiltración entre las incrustaciones inlays en cerómeros y en cerámicas. estudio (in-vitro). Rev. Nac de Odont. 2011; 7 (12): 56-62.

14. Mejía R, Bernardo C, Ruiz X, Espitia J, Moreno J, Montoya A. Evaluación ex vivo de la retención de estructuras en zirconia-ytria de Procera ${ }^{\circledR}$ con diferentes cementos. Rev Fac Odontol Univ Antioq. 2014; 26 (1): 44-61. 
15. Taschner M. Leucite-reinforced glass ceramic inlays luted with self-adhesive resin cement: A 2-year in vivo study. Dent Mater. 2012; 28 (5): 535-40.

16. Pinos M. Microfiltración de estructuras unitarias de dióxido de zirconio con dos tipos de cemento resinoso. [Tesis para especialista en Rehabilitación Oral]. Ecuador: Universidad de Cuenca; 2018.

17. Uludag B, Ozturk N. Microleakage of ceramic inlays luted with differents resin cements and dentin adhesives. J Prosthetic Dent. 2009; 102 (4): 235-41.

18. Korkut $\mathrm{L}$, Cotert $\mathrm{H}$, Kurtulmus $\mathrm{H}$. Marginal, internal fit and microleakage of zirconia infrastructures: an in-vitro study. Oper Dent. 2011; 36 (1): 72-9. 